\title{
Classification of People who Suffer Schizophrenia and Healthy People by EEG Signals using Deep Learning
}

\author{
Carlos Alberto Torres Naira ${ }^{1}$ \\ Escuela Profesional de, Ingeniería de Sistemas \\ Universidad Nacional de, San Agustín de Arequipa \\ Arequipa, Perú
}

\author{
Cristian José López Del Alamo² \\ Escuela Profesional de, Ciencia de la Computación \\ Universidad Nacional de, San Agustín de Arequipa \\ Arequipa, Perú
}

\begin{abstract}
More than 21 million people worldwide suffer from schizophrenia. This serious mental disorder exposes people to stigmatization, discrimination, and violation of their human rights. Different works on classification and diagnosis of mental illnesses use electroencephalogram signals (EEG) because it reflects brain functioning, and how these diseases affect it. Due to the information provided by the EEG signals and the performance demonstrated by Deep Learning algorithms, the present work proposes a model for the classification of schizophrenic and healthy people through EEG signals using Deep Learning methods. Considering the properties of an EEG, high-dimensional and multichannel, we applied the Pearson Correlation Coefficient (PCC) to represent the relations between the channels, this way instead of using the large amount of data that an EEG provides, we used a shorter matrix as an input of a Convolutional Neural Network (CNN). Finally, results demonstrated that the proposed EEG-based classification model achieved Accuracy, Specificity, and Sensitivity of $90 \%, 90 \%$, and $90 \%$, respectively.
\end{abstract}

Keywords-Convolutional Neural Network (CNN); electroencephalography; Electroencephalogram Signals (EEG); deep learning; schizophrenia; classification; Pearson Correlation Coefficient (PCC); Universidad Nacional de San Agustín (UNSA)

\section{INTRODUCTION}

Schizophrenia is a serious mental disorder that is characterized by alterations in thinking, often including psychological experiences such as hearing things or delusions. More than 21 million people worldwide suffer from schizophrenia, of which more than $50 \%$ do not have adequate medical attention [1], being men the ones that suffer it more frequently than women [2].

According to [3], this severe mental disorder can present these symptoms: delusions, hallucinations, disorganized discourse (for example, disaggregation or frequent incoherence), very disorganized or catatonic behavior and negative symptoms (i. e. diminished emotional expression).

The schizophrenics who do not have the proper treatment are more prone to suffer stigmatization, discrimination, and violation of their human rights [1], the life expectancy of people in these patients according to [2], [4] is between 10 to 15 years and their risk of suicide is $10 \%$.

The World Health Organization (WHO) [1] has several programs for the correct treatment of this disorder, which includes pharmacotherapy and psychosocial support, since schizophrenia generally begins in late adolescence or early adulthood [2], the earlier diagnosis is important.

For a schizophrenic to receive adequate treatment, diagnosis is necessary. Due to EEG has unique characteristics, high dimensionality, and variability [5], there are diverse approaches for the diagnosis and treatment of different mental disorders, which work on the data presented by an EEG.

An EEG provides information about the electrical activity of the brain [6], [7], with this information is possible to identify emotions [8], [9], [10], [11], whether a person is healthy or suffer some neurological disease [12], [13], [14], [15], [16], [17].

In different researches, Deep Learning algorithms are used for classification and extraction of characteristics of an EEG, among the most seen are The work of Acharya et al. [6], where the authors used a CNN with 13 layers to detect normal, practical, and seizure classes. In [18] the authors used a CNN with Random Forest and a voting layer to classify highrisk individuals, clinically stable first-episode patients with schizophrenia and healthy controls.

On the other hand, en [19], the authors used CNN to detect and classify segments of EEG which display abnormal brain activity. In [20] was used CNN to classify people who listened and imagined music and compare strategies for learning features. Author in [21] used graph signal representation of an EEG in a CNN, [22] used a CNN to decode and visualize features of an EEG and [23] used CNN to classify between listen to music, reading, and watching augmented reality application, Convolutional Deep Belief Networks [5], Deep Recurrent-Convolutional Neural Networks [24], Deep Neural Networks [9], [15], Restricted Boltzmann Machines [8], Non-Ruinous Autocoder Stacked [25], Deep Belief Network [26].

Observing the performance shown by the Deep Learning algorithms working on EEG signals and the features extracted from them, we propose a method to classify schizophrenic and healthy people. For the other hand, we use PCC to reduce the high-dimensionality and variability presented by each channel of an EGG into a single matrix. This way, the correlation matrix is presented as an input of a $\mathrm{CNN}$ for the classification.

In [11] PCC is used to represent the correlation between the 32 channels of an EEG as a matrix, then the correlation matrix 


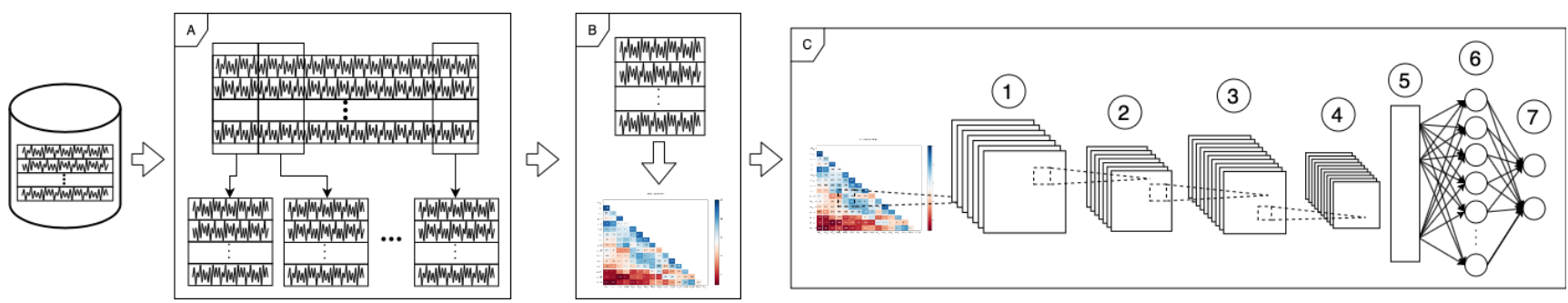

Fig. 1. Methodology proposed (A) Data Segmentation, (B) Pearson Correlation Coefficient and (C) Convolutional Neural Network

is presented as an input for CNNs, obtaining an accuracy of $94.44 \%$ for a CNN with five convolutional layers.

The rest of the paper is organized as follows. In Section II, we give a description of PCC and CNN. In Section III, we describe the data used, the classification procedure and the results obtained. In Section IV, we discuss the results and future work. Finally, in Section V we provide conclusions.

\section{Methodology}

The methodology proposed is shown in Fig. 1 consists of four parts. The first one is how the dataset is composed, which consists of 122880 records per subject (16 channels, and 7680 records per channel). The second part (Section A of Fig. 1) is the Data Segmentation, detailed in subsection II-A, which consists of how the data per subject is segmented and ordered to get a (channels $*$ records) matrix.

The third part (Section B of Fig. 1) is the Pearson Correlation Coefficient, detailed in subsection II-B, is to obtain a matrix that represents the correlation between the channels per subject applying PCC on each (16 channels $* 7680$ records) matrix, the finale matrix is $16 * 16$ dimensions.

The fourth part (Section C of Fig. 1) is the classification with Convolutional Neural Network, detailed in subsection II-C, which consists of using the correlation matrix as an input to a $\mathrm{CNN}$, this way the performance of the $\mathrm{CNN}$ improves performance due to the values of the correlation matrix are between -1 and 1 , instead of the variable values raw EEG signal presents. The CNN consists of two convolutional layers, two max-pooling layers, one fully connected layer, and softmax.

\section{A. Data Segmentation}

Each file that corresponds to a subject contains the 122880 EEG recordings in a column, those 122880 EEG recordings turned as a $16 * 7680$ matrix. 7680 recordings per each channel, taking the first 7680 recordings for the first channel, the second 7680 recordings for the second channel and so on.

Since the length of each channel is too long, we partitioned each EEG recordings matrix per subject into six parts, each one of those partitioned matrices represents ten seconds of each EEG recordings. It means that each $16 * 7680$ matrix turned into six $16 * 1280$ matrices.

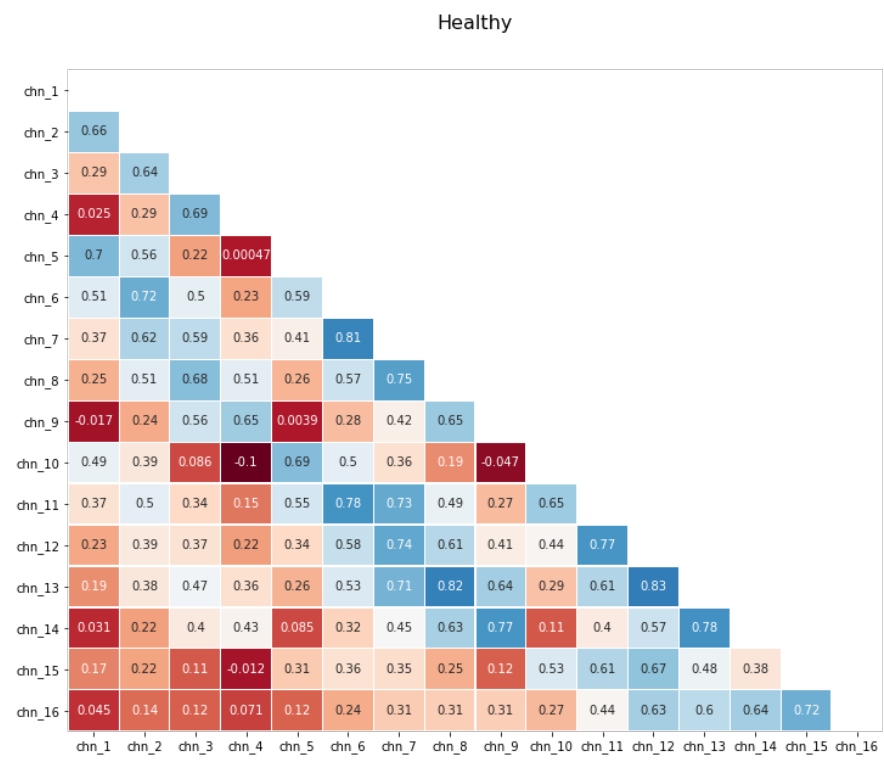

Fig. 2. Heat map of the correlation matrix for a healthy person.

\section{B. Pearson Correlation Coefficient}

The Pearson Correlation Coefficient represents how two variables are related, and in this work, these variables are the signals captured for the electrodes, generating one channel by each of them.

PCC was used to represent how a channel is related to the other ones, this way we turned each sample ( $16 * 1280$ matrix) as a square matrix, which is suitable to be used as an image on a CNN, the Fig. 2 and the Fig. 3 show the correlation matrices of a healthy and schizophrenic person, respectively. 


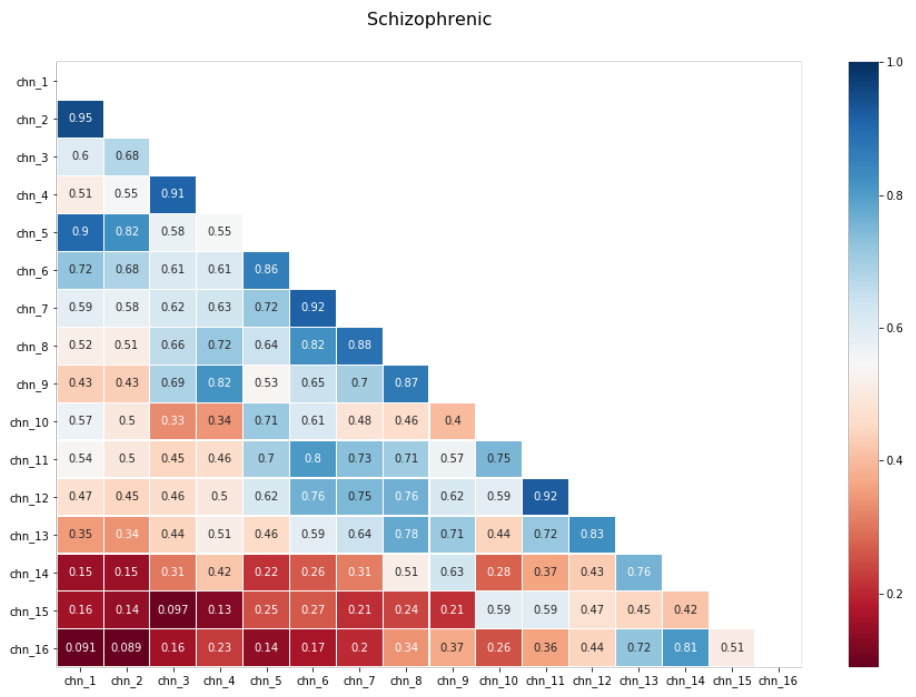

Fig. 3. Heat map of the correlation matrix for a schizophrenic person.

If the two signals are $x$ and $y, \mathrm{PCC}$ is calculated as:

$$
P C C=\frac{\operatorname{cov}(x, y)}{\sigma_{x} \sigma_{y}}
$$

Where $\operatorname{cov}($.$) is the covariance, \sigma_{x}$ and $\sigma_{y}$ the standard deviations of the signals. The value PCC ranges between -1 and 1 , which indicates a perfect negative and perfect positive linear relationship, respectively. So, if PCC is 0 , it means that there is no linear relationship between the two signals.

\section{Convolutional Neural Network}

Once we obtained the correlation matrix per subject, we treated each one as an image for the input of the CNN. work.

In Table I, we detailed the CNN structure used for this

A Stochastic Gradient Descent (SGD) optimizer was used, with a learning rate value of 3 , decay learning value of 0.5 , and momentum of 0.1. And as loss function, Mean Squared Error.

For a better understanding, we define a CNN and its parts below.

CNN is a subset of deep learning [6]. His name comes from the use of convolution filters for complex operations [14], and according to [27], it was inspired biologically by the visual cortex and design at the beginning for image processing.

A typical configuration of a CNN consists of a sequence of convolution and sub-sampled layers. After the last subsampling layer usually follows a series of fully connected layers, this way the 2-D map of characteristics turns into a $1-\mathrm{D}$ vector, and then the classification as traditional Neural Networks (NN) is performed [14].
To avoid overfitting, regularization methods, as dropout, are usually used during training.

CNN comprises three types of layers:

1) Convolutional Layer $A$ set of filters that slide through the input. A Filter is a matrix that convolves the input data and strides control how much the Filter convolves through the length of the input data. The output of the convolution is known as a feature map [6].

2) Pooling Layer Also known as down-sampling layer. Reduce the previous layer neurons output dimension, to relieve the computational intensity and prevent overfitting [6].

Max pooling partitions the input data into rectangles and takes the maximum value from each partition, reducing the output dimension [23].

3) Fully Connected Layer In this layer, all the neurons have a connection with each one of the previous layer neurons, like a hidden layer of a Multilayer Perceptron (MLP).

TABLE I. CNN STRUCTURE

\begin{tabular}{|c|c|}
\hline LAYER & DESCRIPTION \\
\hline $\begin{array}{l}\text { (C.1) CONVOLUTIONAL } \\
\text { LAYER }\end{array}$ & $\begin{array}{l}\text { Layer that uses } 3 * 3 \text { filters that strides } \\
1 * 1 \text { through the input data. }\end{array}$ \\
\hline MAXPOOLING & $\begin{array}{l}\text { Layer that partitions the output data } \\
\text { from the previous layer in } 2 * 2 \text { matrices } \\
\text { and reduces them taking the max value } \\
\text { of each partition. The partitions stride is } \\
2 * 2 \text {. }\end{array}$ \\
\hline $\begin{array}{l}\text { (C.3) CONVOLUTIONAL } \\
\text { LAYER }\end{array}$ & $\begin{array}{l}\text { Layer that uses } 3 * 3 \text { filters that strides } \\
1 * 1 \text { through the input data. }\end{array}$ \\
\hline MAXPOOLING & $\begin{array}{l}\text { Layer that partitions the output data } \\
\text { from the previous layer in } 2 * 2 \text { matrices } \\
\text { and reduces them taking the max value } \\
\text { of each partition. The partitions stride is } \\
2 * 2 \text {. Also, we applied a dropout of } 0.25 \\
\text { to the output data of this layer. }\end{array}$ \\
\hline (C.5) FLATTEN & $\begin{array}{l}\text { Turn the output data from the previous } \\
\text { layer in a } 1-\mathrm{D} \text { vector. }\end{array}$ \\
\hline $\begin{array}{l}\text { (C.6) FULLY CONNECTED } \\
\text { LAYER }\end{array}$ & Layer with 256 neurons. \\
\hline (C.7) SOFTMAX & $\begin{array}{l}\text { Output layer with softmax activation to } \\
\text { the classification. }\end{array}$ \\
\hline
\end{tabular}

It is common to use activation functions after each convolutional layer. An activation function is an operation that maps an output for a group of input.

In this work an activation function is used:

1) Softmax This function computes the probability distribution of the $k$ output classes. The last layer applies this function to predict, which class the input belongs. The output values are between 0 and 1 , and the sum is 1 . 


\section{EXPERIMENTS AND RESULTS}

\section{A. Data Description}

EEG recordings data used for this work, also analyzed in [12], is available at http://brain.bio.msu.ru/eeg_schizophrenia. htm. The recordings belong to two groups. The first one consists of 39 healthy boys (11-13 years old). The second consists of 45 boys (10-14 years old) diagnosed with schizophrenia using clinical interviews at the Research Center for Psychological Disorders of the Russian Academy of Medical Sciences [12]. For all subjects, from 16 channels (electrode positions) the EEG signals were registered, those channels are F7, F3, F4, F8, T3, C3, Cz, C4, T4, T5, P3, Pz, $\mathrm{P} 4, \mathrm{~T} 6, \mathrm{O} 1, \mathrm{O} 2$.

During the recordings, the patients were in a rest state with eyes closed. The signal was recorded for 1 minute at a frequency of $128 \mathrm{~Hz}$, obtaining 7680 records per each channel of a subject.

\section{B. Results}

All this work was developed, executed, and tested using the Google environment Colaboratory, and for the CNN, Keras python library. The EEG signals used in this work come from 45 subjects who suffer schizophrenia and from 39 healthy subjects.

Since the total length of the EEG signals per subject is 60 seconds, we divided those signals into six same length parts. Therefore there will be 270 samples of subjects who suffer schizophrenia, and 234 samples of healthy subjects, each sample with 10 seconds length.

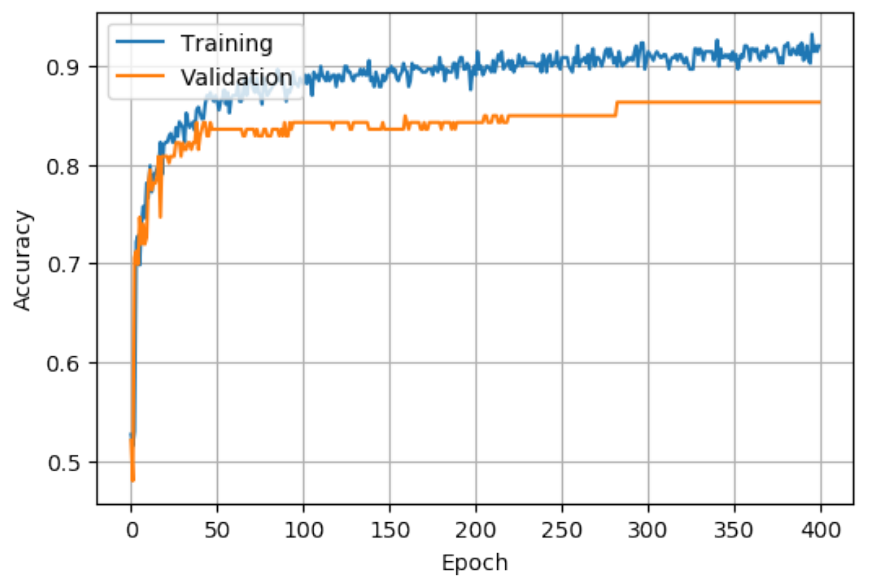

Fig. 4. Accuracy evolution in the training stage.

From all the 504 samples of EEG signals, 484 samples were the training data, and the rest 20 samples the test data.

In the training stage, the number of epochs was set up in 400, and the validation was set up in $30 \%$ for the training data input. Fig. 4 and Fig. 5 show the results in the training stage. For training, the accuracy, and loss values are $92.01 \%$, and 0.076 respectively and for validation, the accuracy and loss values are $86.3 \%$ and 0.094 , respectively.

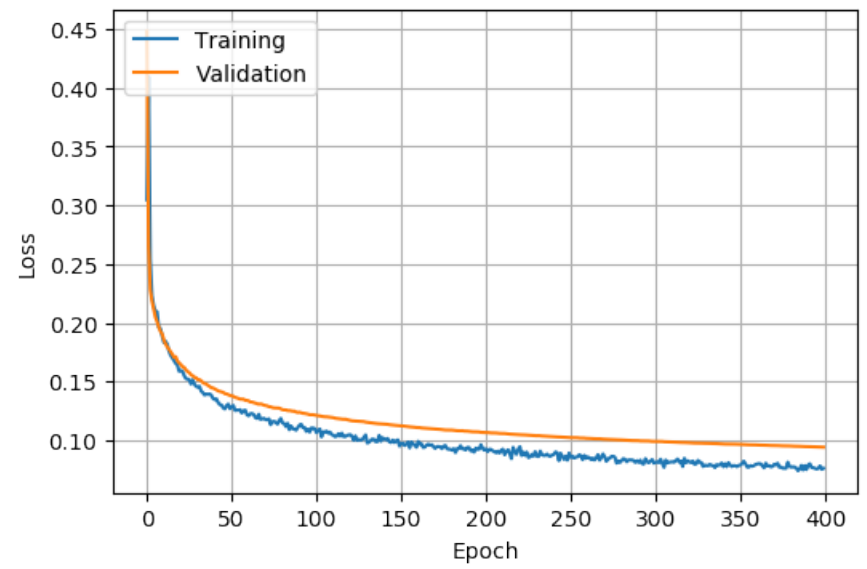

Fig. 5. Loss evolution in the training stage.

The model's performance was calculated in terms of Sensitivity or True Positive Rate (TPR)(2), Specificity or True Negative Rate (TNR)(3) and Accuracy (4) defined as below:

$$
\begin{gathered}
\text { Sensitivity }=\frac{T P}{T P+F N} \\
\text { Specificity }=\frac{T N}{T N+F P} \\
\text { Accuracy }=\frac{T P+T N}{T P+F P+F N+T N}
\end{gathered}
$$

Where TP denotes True Positive (number of Schizophrenia correctly classified as Schizophrenia), FN denotes False Negative (number of Schizophrenia wrongly classified as Healthy), TN denotes True Negative (number of Healthy correctly classified as Healthy), and FP denotes False Positive (number of Healthy wrongly classified Schizophrenia).

The confusion matrix is presented in Table II. It shows that 0.9 of the schizophrenia EEG signals were correctly classified (TPR), and the normal EEG signals reached 0.9 of

\begin{tabular}{|c|c|c|c|}
\hline & \multicolumn{2}{|c|}{ PREDICTED } \\
\hline & & Schizophrenia & Normal \\
\hline \multirow{2}{*}{ ORIGINAL } & Schizophrenia & 0.9 & 0.1 \\
\hline & Normal & 0.1 & 0.9 \\
\hline
\end{tabular}
correct classification (TNR) and accuracy of 0.9 .

TABLE II. CONFUSION MATRIX

\section{Discussion}

The problem of the classification of EEG signals to aid the diagnosis of mental diseases is still open nowadays [12]. Because of this, new researches are developed, trying to 
improve the performance of the previous works.

In this work, a model to classify people who suffer schizophrenia and healthy people by EEG signals using deep learning has demonstrated performance with an accuracy of 0.9 .

Piryatinska et al. [12] presented a model that calculates $\epsilon$-complexity coefficients of the original signal. Then they classified these coefficients with Random Forest (RF) and support vector machine (SVM). Table III shows a comparison between the accuracy reached by [12] and the proposed method, Piryatinska et al. [12] reached an accuracy of 84.5\% applying RF on $\epsilon$-complexity coefficients, and $81.07 \%$ utilizing SVM on $\epsilon$-complexity coefficients, lower than the accuracy of $90 \%$ obtained by our method applying CNN on the channels correlation matrix obtained from raw EEG signals.

TABLE III. COMPARISON OF RESULTS Found IN [12] WITH THE RESULT OF OUR METHOD

\begin{tabular}{|c|c|}
\cline { 2 - 2 } \multicolumn{1}{c|}{} & Accuracy \\
\hline$\epsilon$-complexity coefficients with RF & $84.5 \%$ \\
\hline$\epsilon$-complexity coefficients with SVM & $81.07 \%$ \\
\hline Our method & $90 \%$ \\
\hline
\end{tabular}

\section{CONCLUSION}

In conclusion, we proposed a model that uses PCC to obtain the correlation matrix of the EEG signal and present it to a $\mathrm{CCN}$ architecture, to classify people who suffer schizophrenia and healthy people from publicly available data. The proposed methodology obtained an accuracy of $90 \%$, with a specificity of $90 \%$ and a sensitivity of $90 \%$. The performance of the proposed method is better than the existing work that used the same data.

Increasing the amount of data, the performance of the proposed method can be better.

\section{ACKNOWLEDGMENT}

The authors would like to thank the support and subvention of the UNIVERSIDAD NACIONAL DE SAN AGUSTÍN DE AREQUIPA with contract $\mathrm{N}^{\mathrm{O}}$ TP-5-2018-UNSA.

\section{REFERENCES}

[1] W. H. Organization, "Schizophrenia," https://www.who.int/en/newsroom/fact-sheets/detail/schizophrenia, 2018.

[2] J. M. Lyness, "Psychiatric Disorders in Medical Practice," Goldman's Cecil Medicine: Twenty Fourth Edition, vol. 2, pp. 2236-2245, 2011. [Online]. Available: http://dx.doi.org/10.1016/B978-1-4377-1604-7. 00404-8

[3] Asociación Americana de Psiquiatría, "Guia De Consulta De Los Criterios Diagnóticos Del Dsm-5,” pp. 107-110, 2014. [Online]. Available: www.psych.org

[4] M. A. Nihart, "The neurobiology of schizophrenia," Journal of the American Psychiatric Nurses Association, vol. 2, no. 5, pp. 61-82, 2016.

[5] Y. Ren and Y. Wu, "Convolutional deep belief networks for feature extraction of EEG signal," 2014 International Joint Conference on Neural Networks (IJCNN), pp. 2850-2853, 2014. [Online]. Available: http://ieeexplore.ieee.org/lpdocs/epic03/wrapper. htm?arnumber $=6889383$
[6] U. R. Acharya, S. L. Oh, Y. Hagiwara, J. H. Tan, and H. Adeli, "Deep convolutional neural network for the automated detection and diagnosis of seizure using eeg signals," Computers in biology and medicine, vol. 100, pp. 270-278, 2018.

[7] F. Lotte, L. Bougrain, A. Cichocki, M. Clerc, M. Congedo, A. Rakotomamonjy, and F. Yger, "A review of classification algorithms for eegbased brain-computer interfaces: a 10 year update," Journal of neural engineering, vol. 15 , no. 3, p. 031005, 2018.

[8] Y. Gao, H. J. Lee, and R. M. Mehmood, "Deep learninig of eeg signals for emotion recognition," in Multimedia \& Expo Workshops (ICMEW), 2015 IEEE International Conference on. IEEE, 2015, pp. 1-5.

[9] A. Frydenlund and F. Rudzicz, "Emotional affect estimation using video and eeg data in deep neural networks," in Canadian Conference on Artificial Intelligence. Springer, 2015, pp. 273-280.

[10] S. Jirayucharoensak, S. Pan-Ngum, and P. Israsena, "Eeg-based emotion recognition using deep learning network with principal component based covariate shift adaptation," The Scientific World Journal, vol. 2014, 2014.

[11] S.-E. Moon, S. Jang, and J.-S. Lee, "Convolutional neural network approach for eeg-based emotion recognition using brain connectivity and its spatial information," in 2018 IEEE International Conference on Acoustics, Speech and Signal Processing (ICASSP). IEEE, 2018, pp. 2556-2560.

[12] A. Piryatinska, B. Darkhovsky, and A. Kaplan, "Binary classification of multichannel-eeg records based on the $\epsilon$-complexity of continuous vector functions," Computer methods and programs in biomedicine, vol. 152, pp. 131-139, 2017.

[13] B. Pandey and D. Kundra, "Diagnosis of eeg-based diseases using data mining and case-based reasoning," International Journal of Intelligent Systems Design and Computing, vol. 1, no. 1-2, pp. 43-55, 2017.

[14] D. Ravì, C. Wong, F. Deligianni, M. Berthelot, J. Andreu-Perez, B. Lo, and G.-Z. Yang, "Deep learning for health informatics," IEEE journal of biomedical and health informatics, vol. 21, no. 1, pp. 4-21, 2016.

[15] A. S. Heinsfeld, A. R. Franco, R. C. Craddock, A. Buchweitz, and F. Meneguzzi, "Identification of autism spectrum disorder using deep learning and the abide dataset," NeuroImage: Clinical, vol. 17, pp. 1623, 2018.

[16] H. Liu, T. Zhang, Y. Ye, C. Pan, G. Yang, J. Wang, and R. C. Qiu, "A data driven approach for resting-state eeg signal classification of schizophrenia with control participants using random matrix theory," arXiv preprint arXiv:1712.05289, 2017.

[17] M. Shim, H.-J. Hwang, D.-W. Kim, S.-H. Lee, and C.-H. Im, "Machinelearning-based diagnosis of schizophrenia using combined sensor-level and source-level eeg features," Schizophrenia research, vol. 176, no. 2-3, pp. 314-319, 2016.

[18] L. Chu, R. Qiu, H. Liu, Z. Ling, and X. Shi, "Individual Recognition in Schizophrenia using Deep Learning Methods with Random Forest and Voting Classifiers: Insights from Resting State EEG Streams," arXiv preprint arXiv:1707.03467, pp. 1-7, 2017.

[19] A. Ganta, "Detection and Classification of Epileptiform Transients in EEG Signals Using Convolution Neural Network," 2017.

[20] S. Stober, A. Sternin, A. M. Owen, and J. A. Grahn, "Deep feature learning for eeg recordings," arXiv preprint arXiv:1511.04306, 2015.

[21] S. Jang, S.-E. Moon, and J.-S. Lee, "Graph signal representation of eeg for graph convolutional neural network," 2018.

[22] R. T. Schirrmeister, J. T. Springenberg, L. D. J. Fiederer, M. Glasstetter, K. Eggensperger, M. Tangermann, F. Hutter, W. Burgard, and T. Ball, "Deep learning with convolutional neural networks for eeg decoding and visualization," Human brain mapping, vol. 38 , no. 11, pp. 53915420, 2017.

[23] S. Sarkar, K. Reddy, A. Dorgan, C. Fidopiastis, and M. Giering, "Wearable eeg-based activity recognition in phm-related service environment via deep learning," Int. J. Progn. Health Manag, vol. 7, pp. 1-10, 2016.

[24] P. Bashivan, I. Rish, M. Yeasin, and N. Codella, "Learning Representations from EEG with Deep Recurrent-Convolutional Neural Networks," arXiv preprint arXiv:1511.06448, pp. 1-15, 2015. [Online]. Available: http://arxiv.org/abs/1511.06448 
[25] Z. Yin and J. Zhang, "Cross-session classification of mental workload levels using eeg and an adaptive deep learning model," Biomedical Signal Processing and Control, vol. 33, pp. 30-47, 2017.

[26] F. Movahedi, J. L. Coyle, and E. Sejdić, "Deep belief networks for electroencephalography: A review of recent contributions and future outlooks," IEEE journal of biomedical and health informatics, vol. 22, no. 3, pp. 642-652, 2017.

[27] S. Vieira, W. H. Pinaya, and A. Mechelli, "Using deep learning to investigate the neuroimaging correlates of psychiatric and neurological disorders: Methods and applications," Neuroscience \& Biobehavioral Reviews, vol. 74, pp. 58-75, 2017. 